\title{
Some Backward in Time Results for Thermoelastic Dipolar Structures
}

\author{
Marin I. Marin ${ }^{1 *}$, Ibrahim A. Abbas ${ }^{2}$, Sorin Vlase ${ }^{3}$ and Eduard-Marius Craciun ${ }^{4}$ \\ ${ }^{1}$ Department of Mathematics and Computer Science, Transilvania University of Brasov, Brasov, Romania, ${ }^{2}$ Department of \\ Mathematics, Faculty of Science, Sohag University, Sohag, Egypt, ${ }^{3}$ Department of Mechanical Engineering, Transilvania \\ University of Brasov, Brasov, Romania, ${ }^{4}$ Faculty of Mechanical, Industrial and Maritime Engineering, Ovidius University of \\ Constanta, Constanta, Romania
}

In our study, we define the linear backward in time problem for a thermoelastic body with a dipolar structure. In our first main result, we prove the uniqueness of the solution for this problem. Afterward, the issue of locating solutions of the backward in time problem in time will be approached as a consequence of the uniqueness result. In fact, we address the question of the impossibility of locating the solution of this problem in time.

Keywords: body with dipolar structure, backward in time problem, uniqueness result, localization in time of solution, thermoelasticity

\section{OPEN ACCESS}

Edited by:

Dumitru Baleanu,

University of Craiova, Romania

Reviewed by:

Amin Jajarmi,

University of Bojnord, Iran

Babak Shiri,

Neijiang Normal University, China

*Correspondence:

Marin I. Marin

m.marin@unitbv.ro

Specialty section:

This article was submitted to

Mathematical Physics,

a section of the journal

Frontiers in Physics

Received: 22 December 2019

Accepted: 13 February 2020

Published: 10 March 2020

Citation:

Marin MI, Abbas IA, Vlase S and Craciun E-M (2020) Some Backward in Time Results for Thermoelastic Dipolar Structures. Front. Phys. 8:41.

doi: 10.3389/fphy.2020.00041

\section{INTRODUCTION}

Our study is dedicated to a dipolar structure for a thermoelastic body. This kind of material falls within a more general theory, namely that of microstructure, initiated by Eringen (see, for instance, $[1,2])$. The importance of the dipolar structure can be deduced from the great number of studies that have appeared on this subject; see for instance [3-7]. Therefore, our present study is a continuation of research in this domain.

Another kind of microstructure is also obtained by taking into account voids in materials, starting with the paper of Nunziato and Cowin [8]. Since then, the number of studies on this topic has become impressive. Here, we mention just some of these: [9-16] It is considered that the initiator of backward in time problems is Serrin, who approached these kinds of problems by taking into account the Navier-Stokes equations (see [17]). Some results of uniqueness for the forward in time problem can be found in the study [17]. More recently, the number of articles related to the backward in time problem has significantly increased; we can recommend [18-27]. As a particular observation, the results of Quintanilla [25] improve on the studies of Ciarletta [23] and of Ciarletta and Chirita [24]. Other studies by Quintanilla solve some questions regarding location in time when considering the solutions for backward in time problems, even in the theory of thermoelastic porous bodies and of thermoelasticity of Green and Naghdi [26, 27]. Iovane and Passarella [28] also approached elastic bodies with voids. Passarella and Tibullo [29] considered the backward in time problem for thermo-microstretch elastic bodies. Other results regarding bodies with microstructure can be found in Marin et al. [30], Marin and Florea [31], and Marin [32, 33]. We should point out that our idea to consider the backward in time problem in the context of the theory of dipolar bodies was stimulated by the paper of Quintanilla and Straughan [34]. So far, no study has addressed the problem of the localization in time of solutions for the mixed problem in the context of thermoelastic dipolar solids. Inspired by articles [35-37], in our future studies, we will try to generalize the results of this study in the sense that we will try to replace the common derivative with a fractional one. Now we set out the structure of our paper. First, 
in section 2, we detail the system of the main differential equations. After that, as usual, we add the boundary conditions. The mixed-value problem obtained is a final value problem. Using an accessible transformation, the final problem is replaced by an initial value problem. For this last problem, we prove the uniqueness of the solution. Afterward, the issue of locating solutions of the backward in time problem in time will be approached as a consequence of the result of uniqueness. In fact, we address the question of the impossibility of locating the solution of this problem in time.

\section{PRELIMINARY EQUATIONS AND CONDITIONS}

As already seen, our study is dedicated to a thermoelastic dipolar body. We consider an anisotropic and non-homogeneous body that occupies a regular region $D$, included in the Euclidean space $R^{3}$. The boundary of the domain $D$ is a piecewise regular surface, denoted by $\partial D$. As usual, we use the notation $\bar{D}$ for the closure of the domain $D$, and we have $\bar{D}=D \cup \partial D$. We will use an orthonormal system of references and suppose that the tensors and vectors have components with indices over 1,2,3. The convention for summation in case of a repeated index is used, and the derivation operations are correspondingly defined. So, a partial derivative with respect to a spatial coordinate is designated by a subscript preceded by a comma. A superposed dot is used to designate the derivative with regards to $t$, the time variable. All functions used in our study are assumed to be as regular as necessary. Sometimes, the dependence of functions regarding the time or spatial independent variables is omitted, of course, when there is no possibility of confusion.

In order to describe the evolution of a dipolar thermoelastic body, we will use the following variables:

$$
u_{i}(t, x), \phi_{i j}(t, x), \theta(t, x),(t, x) \in\left[0, t_{0}\right) \times D,
$$

where we denote the displacement vector field by $u=\left(u_{i}\right)$. By $\phi=\left(\phi_{i j}\right)$, we denote the dipolar displacement tensor field.

Using the above variables $u_{i}(x, t), \phi_{i j}(x, t)$, we will introduce the components of the tensors of strain, namely $\varepsilon_{i j}, \kappa_{i j}$, and $\chi_{i j k}$, as follows:

$$
2 \varepsilon_{i j}=u_{j, i}+u_{i, j}, \kappa_{i j}=u_{j, i}-\phi_{i j}, \chi_{i j k}=\phi_{i j, k} .
$$

We must specify that our further considerations refer to the linear theory; as such, we can consider Helmholtz's free energy as a quadratic form with regard to all its independent variables. Let us denote by $W$ the Helmholtz's free density of energy in the initial configuration. As a consequence, according to the principle of energy conservation, we will expand in series the function $W$ and retain only the terms until the second order. If we take into account that the initial state (the reference state) is supposed free of charges, we are led to the conclusion that the Helmholtz's energy per unit mass has the following expression:

$$
W=\frac{1}{2} A_{i j m n} \varepsilon_{i j} \varepsilon_{m n}+D_{i j m n} \varepsilon_{i j} \kappa_{m n}+F_{i j m n r} \varepsilon_{i j} \chi_{m n r}
$$

$$
\begin{aligned}
& +\frac{1}{2} B_{i j m n} \kappa_{i j} \kappa_{m n}+G_{i j m n r} \kappa_{i j} \chi_{m n r}+\frac{1}{2} C_{i j k m n r} \chi_{i j k} \chi_{m n r} \\
& -a_{i j} \varepsilon_{i j} \theta-b_{i j} \kappa_{i j} \theta-c_{i j k} \chi_{i j k} \theta-\frac{1}{2} c \theta^{2} .
\end{aligned}
$$

If we substitute the Helmholtz's energy into the entropy production inequality, we obtain a relation from which the equations of motion are deduced. Also, the same relation can be used to deduce the constitutive equations, as it is known that the constitutive equations give the expression of the stress tensors as functions of the strain tensors and some constants of the material. In what follows, we will use the notations $\tau_{i j}, \eta_{i j}$, and $\mu_{i j k}$ for the stress tensors. In our case the relationships that describe the connections $\tau_{i j}, \eta_{i j}, \mu_{i j k}$, and the tensors $\varepsilon_{i j}, \kappa_{i j}, \chi_{i j k}$ are the constitutive equations.

Inspired by the procedure used by Green and Rivlin in the paper [6], we adopt a similar technique so that by taking into account the Helmholtz's energy (3), we are led to the following constitutive equations:

$$
\begin{aligned}
\tau_{i j} & =\frac{\partial W}{\partial \varepsilon_{i j}}=A_{i j m n} \varepsilon_{m n}+D_{m n i j} \kappa_{m n}+F_{m n r i j} \chi_{m n r}-a_{i j} \theta, \\
\eta_{i j} & =\frac{\partial W}{\partial \kappa_{i j}}=D_{i j m n} \varepsilon_{m n}+B_{i j m n} \kappa_{m n}+G_{i j m n r} \chi_{m n r}-b_{i j} \theta, \\
\mu_{i j k} & =\frac{\partial W}{\partial \chi_{i j k}}=F_{i j k m n} \varepsilon_{m n}+G_{m n i j k} \kappa_{m n}+C_{i j k m n r} \chi_{m n r}-c_{i j k} \theta, \\
\eta & =-\frac{\partial W}{\partial \theta}=a_{i j} \varepsilon_{i j}+b_{i j} \kappa_{i j}+c_{i j k} \chi_{i j k}+c \theta,
\end{aligned}
$$

where we use the notation $\eta$ to designate the entropy (per unit mass).

We will consider that the above equations take place in a cylinder $\left[0, t_{0}\right) \times D$.

Denoting the components of the heat flux vector by $q_{i}$, we can deduce a constitutive relation similar to that from the classical theory, namely

$$
q_{i}=K_{i j} \theta_{, j}
$$

where the thermal conductivity tensor is denoted by $K_{i j}$ and it is assumed that $K_{i j}$ is a symmetric tensor.

The differential equations that govern the thermoelasticity of dipolar bodies, obtained as in Mindlin [5] and Green and Rivlin [6], are:

- the equations of motion:

$$
\begin{aligned}
& \left(\tau_{i j}+\eta_{i j}\right)_{, j}+\rho f_{i}=\rho \ddot{u}_{i}, \\
& \mu_{i j k, i}+\eta_{j k}+\rho g_{j k}=I_{k r} \ddot{\phi}_{j r} ;
\end{aligned}
$$

- the equation of energy:

$$
\rho T_{0} \dot{\eta}=q_{i, i}+\rho r
$$

In the preceding equations, we used some notations with the following signification: $\rho$ - the constant mass density; $I_{i j}$ - the tensor of microinertia, which is a symmetric tensor; $k$ - a scalar for the intrinsic inertia; $\varepsilon_{i j}, \kappa_{i j}, \chi_{i j k}$ - the strain tensors; $\tau_{i j}, \eta_{i j}, \mu_{i j k}$ 
- the stress tensors; $f_{i}$ - the body forces; $g_{j k}$ - the dipolar charges; $A_{i j m n}, B_{i j m n}, \ldots, a_{i j}$ - the coefficients that characterize the properties of the material with regards to elasticity. Clearly, from

(3) we can deduce the following symmetry relations:

$$
\begin{aligned}
& A_{i j m n}=A_{j i m n}=A_{m n i j}, B_{i j m n}=B_{m n i j}, a_{i j}=a_{j i}, \\
& C_{i j k m n r}=C_{m n r i j k}, F_{i j k m n}=F_{i j k n m}, D_{i j m n}=D_{i j n m} .
\end{aligned}
$$

Consider the Equations (6) and (7), in the particular case where there are no supply terms and taking into account the kinematic equations (2) and the constitutive relations (4) and (5). Then, from the equations of motion and the energy equations, we obtain the following system of equations:

$$
\begin{aligned}
\rho \ddot{\mathrm{u}}_{i}= & {\left[\left(C_{i j m n}+G_{i j m n}\right) \mathrm{u}_{n, m}\right.} \\
& +\left(G_{m n i j}+B_{i j m n}\right)\left(\mathrm{u}_{n, m}-\phi_{m n}\right)+ \\
& \left.+\left(F_{m n r i j}+D_{i j m n r}\right) \phi_{n r, m}-\left(a_{i j}+b_{i j}\right) \theta\right]_{, j}, \\
I_{k r} \ddot{\phi}_{j r}= & {\left[F_{i j k m n} \mathrm{u}_{n, m}+D_{m n i j k}\left(\mathrm{u}_{n, m}-\phi_{m n}\right)+A_{i j k m n r} \phi_{n r, m}\right.} \\
& \left.-c_{i j k} \theta\right]_{, i}+G_{j k m n} \mathrm{u}_{m, n} \\
& +B_{j k m n}\left(\mathrm{u}_{n, m}-\phi_{m n}\right)+D_{j k m n r} \phi_{n r, m}-b_{j k} \theta, \\
K_{i j}\left(\theta_{, j}\right)_{, i}= & -T_{0}\left[a_{i j} \dot{u}_{i, j}+b_{i j}\left(\dot{\mathrm{u}}_{j, i}-\dot{\phi}_{i j}\right)+c_{i j k} \dot{\phi}_{i j, k}+c \dot{\theta}\right],
\end{aligned}
$$

which take place in the interval $(-\infty, 0]$.

We also suppose that the Equations (2), (4) take place in the interval $(-\infty, 0]$.

We now want to define the surface tractions vector of components $t_{i}$, a surface couple tensor of components $\mu_{j k}$ and the flux of heat denoted by $q$. To this end, we need the unit normal to the border $\partial D$, of components $n_{i}$, outward oriented. These have the following expressions:

$$
t_{i}=\left(\tau_{i j}+\eta_{i j}\right) n_{j}, \mu_{j k}=\mu_{i j k} n_{i}, q=q_{i} n_{i},
$$

and are defined at regular points of the border $\partial D$.

Having these tractions, we can now consider the following boundary data, in their homogeneous form:

$$
\begin{array}{r}
u_{i}(x, t)=0,(x, t) \in \partial D_{u} \times(-\infty, 0], t_{i}=0, \\
(x, t) \in \partial D_{u}^{c} \times(-\infty, 0], \phi_{i j}(x, t)=0,(x, t) \in \partial D_{\phi} \\
\times(-\infty, 0], m_{j k}=0,(x, t) \in \partial D_{\phi}^{c} \\
\times(-\infty, 0], \theta(x, t)=0,(x, t) \in \partial D_{\theta} \times(-\infty, 0], \\
q=0,(x, t) \in \partial D_{\theta}^{c} \times(-\infty, 0],
\end{array}
$$

where the subsets $\partial D_{u}, \partial D_{\phi}, \partial D_{\theta}$ and their complements $\partial D_{\mathcal{u}}^{c}, \partial D_{\phi}^{c}, \partial D_{\theta}^{c}$ are surfaces from the boundary $\partial D$ with the following properties:

$$
\begin{gathered}
\partial \bar{D}_{u} \cup \partial D_{u}^{c}=\partial \bar{D}_{\phi} \cup \partial D_{\phi}^{c}=\partial \bar{D}_{\theta} \cup \partial D_{\theta}^{c}=\partial D, \\
\partial D_{u} \cap \partial D_{u}^{c}=\partial D_{\phi} \cap \partial D_{\phi}^{c}=\partial D_{\theta} \cap \partial D_{\theta}^{c}=\emptyset .
\end{gathered}
$$

The mixed problem will be complete if we add the final restrictions:

$$
u_{i}(x, 0)=u_{i}^{0}(x), \dot{u}_{i}(x, 0)=u_{i}^{1}(x), \theta(x, 0)=\theta^{0}(x),
$$

$$
\phi_{i j}(x, 0)=\phi_{i j}^{0}(x), \dot{\phi}_{i j}(x, 0)=\phi_{i j}^{1}(x)
$$

which take place on the closed domain $\bar{D}$. Here $u_{i}^{0}(x)$, $u_{i}^{1}(x), \phi_{i j}^{0}(x), \phi_{i j}^{1}(x)$, and $\theta^{0}(x)$ are given and are continuous functions. Furthermore, we must suppose that these functions are compatible with the restrictions (11) on the appropriate sub-surfaces of $\partial D$.

The internal energy $\Psi$ will be considered as a quadratic form with the following representation

$$
\begin{aligned}
\Psi & =\frac{1}{2} A_{i j m n} \varepsilon_{i j} \varepsilon_{m n}+D_{i j m n} \varepsilon_{i j} \kappa_{m n}+F_{i j m n r} \varepsilon_{i j} \chi_{m n r}+ \\
& +\frac{1}{2} B_{i j m n} \kappa_{i j} \kappa_{m n}+G_{i j m n r} \kappa_{i j} \chi_{m n r}+\frac{1}{2} C_{i j k m n r} \chi_{i j k} \chi_{m n r}
\end{aligned}
$$

Let us denote by $\mathcal{P}$ our boundary-final value problem, which consists of the system of basic equations (9), the final conditions (12), and the boundary data (11).

The results we want to expose will only be possible if certain restrictions are met, imposed on all the functions we will deal with.

First, we denote by $J_{m}(x)$ the minimum eigenvalue of the tensor of inertia $I_{i j}(x)$, and we need to suppose that $J_{m}$ is a continuous function. Also, the density $\rho$ and the constitutive coefficients are assumed be functions of class $C^{1}(D)$. Also, we must suppose that:

(a) $\rho(x) \geq a_{1}, J_{m}(x) \geq a_{2}, c(x) \geq a_{3}$, where the real constants $a_{1}, a_{2}, a_{3}$ are positive;

(b) $K_{i j}$ is a positive definite tensor;

(c) the quadratic form $\Psi$ is positive definite.

As a consequence of hypothesis (b), we can deduce the inequalities:

$$
K_{m} \theta_{, i} \theta_{, j} \leq K_{i j} \theta_{, i} \theta_{, j} \leq K_{M} \theta_{, i} \theta_{, j}
$$

where $K_{m}$ and $K_{M}$ are two positive constants.

If we take into account hypothesis (c), then we can determine two positive numbers $M_{1}$ and $M_{2}$ to satisfy the double inequality that follows:

$$
\begin{aligned}
& \frac{M_{1}}{2}\left(\varepsilon_{i j} \varepsilon_{i j}+\kappa_{i j} \kappa_{i j}+\chi_{i j k} \chi_{i j k}\right) \leq \Psi \\
& \leq \frac{M_{2}}{2}\left(\varepsilon_{i j} \varepsilon_{i j}+\kappa_{i j} \kappa_{i j}+\chi_{i j k} \chi_{i j k}\right) .
\end{aligned}
$$

The above restrictions are commonly imposed in the mechanics of solids, so they cannot be considered as very restrictive.

Now our concerns are for transforming the boundaryfinal value problem $\mathcal{P}$ into an initial-boundary values problem denoted by $\mathcal{P}^{\prime}$. To this aim, we will make a convenient transformation of variables. So, in this regard, if $h$ is the notation for a generic function, we set $h^{\prime}\left(t^{\prime}\right)=h(t)$, for $t^{\prime}=-t$. However, we will renounce to the sign "prime" so as not to complicate the writing. As such, the above problem $\mathcal{P}^{\prime}$ consists of the following restrictions and equations:

- the equations of motion $(9)_{1}$ and $(9)_{2}$, which take place in the cylinder $D \times[0, \infty)$; 
- the equation of energy:

$$
\begin{array}{r}
K_{i j}\left(\theta_{j, j}\right)_{, i}=T_{0}\left[a_{i j} \dot{\mathrm{u}}_{i, j}+b_{i j}\left(\dot{\mathrm{u}}_{j, i}-\dot{\phi}_{i j}\right)+c_{i j k} \dot{\phi}_{i j, k}+c \dot{\theta}\right], \\
\operatorname{in~} D \times[0, \infty) ;
\end{array}
$$

- the kinematic equations (2), which take place in the cylinder $D \times[0, \infty)$;

- the constitutive conditions (4), which take place in the cylinder $D \times[0, \infty)$;

- the initial restrictions (11), which take place in $\bar{D}$;

- the conditions to the limit:

$$
\begin{aligned}
\mathrm{u}_{i}(x, t)=0,(x, t) \in \partial D_{u} \times[0, \infty), \\
t_{i}=0,(x, t) \in \partial D_{u}^{c} \times[0, \infty), \\
\phi_{i j}(x, t)=0,(x, t) \in \partial D_{\phi} \times[0, \infty), \\
m_{j k}=0,(x, t) \in \partial D_{\phi}^{c} \times[0, \infty), \\
\theta(x, t)=0,(x, t) \in \partial D_{\theta} \times[0, \infty), \\
q=0,(x, t) \in \partial D_{\theta}^{c} \times[0, \infty) .
\end{aligned}
$$

\section{MAIN RESULTS}

At the beginning of this section we will establish some identities, of integral type, with regards to the solutions $\mathbf{u}=\left(u_{i}, \phi_{i j}, \theta\right)$ of the above mixed problem $\mathcal{P}^{\prime}$. The important results of our study will be based on these identities.

Proposition 1. If $\mathbf{u}=\left(u_{i}, \phi_{i j}, \theta\right)$ is a solution of our above problem $\mathcal{P}^{\prime}$, then the following identity is satisfied

$$
\begin{aligned}
& \frac{1}{2} \int_{B}\left[\left(\rho \dot{u}_{i}(t) \dot{u}_{i}(t)+I_{j k} \dot{\phi}_{j m}(t) \dot{\phi}_{k m}(t)\right)+2 \Psi(t)+c \theta^{2}(t)\right] d V= \\
& =\int_{B}\left[\rho \dot{u}_{i}(0) \dot{u}_{i}(0)+I_{j k} \dot{\phi}_{j m}(0) \dot{\phi}_{k m}(0)+\Psi(0)+\frac{1}{2} c \theta^{2}(0)\right](18) \\
& d V++\int_{0}^{t} \int_{D} \frac{1}{T_{0}} K_{i j} \theta_{, i}(\tau) \theta_{, j}(\tau) d V d \tau, \forall t \in[0, \infty) .
\end{aligned}
$$

Proof. If we consider the kinematic equations (2) and take into account the equations of motion $(9)_{1}$ and $(9)_{2}$, then the following identity is obtained:

$$
\begin{aligned}
& \frac{1}{2} \frac{\partial}{\partial t}\left(\rho \dot{\mathrm{u}}_{i}(t) \dot{\mathrm{u}}_{i}(t)+I_{j k} \dot{\phi}_{j m}(t) \dot{\phi}_{k m}(t)\right)= \\
= & {\left[\left(\tau_{i j}+\eta_{i j}\right) \dot{\mathrm{u}}_{j}+\mu_{i j k} \dot{\phi}_{j k}\right]_{, i}-\left(\tau_{i j} \dot{\varepsilon}_{i j}+\eta_{i j} \dot{\kappa}_{i j}+\mu_{i j k} \dot{\chi}_{i j k}\right) . }
\end{aligned}
$$

Now, we consider the equations (4) and the form of the energy density $\Psi$, defined in (13). Taking into account the relations of symmetry (8), we find that the parenthesis at the end of the identity (19) receives the following form

$$
\begin{aligned}
\left(\tau_{i j} \dot{\varepsilon}_{i j}+\eta_{i j} \dot{\kappa}_{i j}+\mu_{i j k} \dot{\chi}_{i j k}\right) & =\frac{\partial}{\partial t}\left(\Psi+\frac{1}{2} c \theta^{2}\right)+\left(\frac{1}{T_{0}} q_{j} \theta\right)_{, j} \\
& -\frac{1}{T_{0}} K_{i j} \theta_{, i} \theta_{, i} .
\end{aligned}
$$

Now we introduce Equation (20) into the equality (19) and we integrate the resulting identity over the domain $[0, t] \times D$. It remains only to use the divergence theorem and to take into account the homogeneous conditions data to the limit (17), such that we obtain the identity (18), as such, so that the proof of Proposition 1 is finished.

A complement of the equality (18) is the identity that follows:

$$
\begin{aligned}
& \frac{1}{2} \int_{B}\left[\left(\rho \dot{u}_{i}(t) \dot{u}_{i}(t)+I_{j k} \dot{\phi}_{j m}(t) \dot{\phi}_{k m}(t)\right)+2 \Psi(t)-c \theta^{2}(t)\right] d V= \\
& =\int_{B}\left[\rho \dot{u}_{i}(0) \dot{u}_{i}(0)+I_{j k} \dot{\phi}_{j m}(0) \dot{\phi}_{k m}(0)+\Psi(0)\right. \\
& \left.-\frac{1}{2} c \theta^{2}(0)\right] d V- \\
& -\int_{0}^{t} \int_{D}\left\{\dot{u}_{i}(\tau)\left[\left(a_{i j}+b_{j i}\right) \theta(\tau)\right]_{, j}+\dot{\phi}_{i j}(\tau)\left[c_{i j k} \theta(\tau)\right]_{, k}\right. \\
& \left.--b_{i j} \dot{\phi}_{i j}(\tau) \theta(\tau)+\frac{1}{T_{0}} K_{i j} \theta_{, i}(\tau) \theta_{, j}(\tau)\right\} \\
& d V d \tau, \forall t \in[0, \infty),
\end{aligned}
$$

which can be proven by using a procedure similar to the one used to prove Proposition 1.

Let us enter the notation

$$
\begin{aligned}
2 F(x, y)= & A_{i j m n} \varepsilon_{i j}(x) \varepsilon_{m n}(y)+D_{i j m n}\left[\varepsilon_{i j}(x) \kappa_{m n}(y)\right. \\
& \left.+\varepsilon_{i j}(y) \kappa_{m n}(x)\right]++F_{i j m n r}\left[\varepsilon_{i j}(x) \chi_{m n r}(y)\right. \\
& \left.+\varepsilon_{i j}(y) \chi_{m n r}(x)\right] \\
& +B_{i j m n} \kappa_{i j}(x) \kappa_{m n}(y) \\
& ++G_{i j m n r}\left[\kappa_{i j}(x) \chi_{m n r}(y)+\kappa_{i j}(y) \chi_{m n r}(x)\right] \\
& +C_{i j k m n r} \chi_{i j k}(x) \chi_{m n r}(y),
\end{aligned}
$$

in order to simplify the writing.

Then, from (22) it is easy to obtain the symmetry property of the quadratic form $F(x, y)$ :

$$
F(x, y)=F(y, x)
$$

Here, we considered the relations of symmetry (8). Also, if we analyze Equation (13), then by a simple substitution in (22), we are led to the identity

$$
F(\tau, \tau)=\Psi(\tau)
$$

These above considerations will help us to obtain an analogous identity to those from (18) but considered in the particular case of null initial data.

Proposition 2. Let $\left(u_{i}, \phi_{i j}, \theta\right)$ be a solution of the backward in time problem, $\mathcal{P}^{\prime}$, corresponding to zero initial conditions. Then, for all $t \in[0, \infty)$, the following equality is satisfied:

$$
\begin{array}{r}
\frac{1}{2} \int_{B}\left[\left(\rho \dot{u}_{i}(t) \dot{u}_{i}(t)+I_{j k} \dot{\phi}_{j m}(t) \dot{\phi}_{k m}(t)\right)-c \theta^{2}(t)\right] d V= \\
=\int_{B}\left[\frac{1}{2} A_{i j m n} \varepsilon_{i j}(t) \varepsilon_{m n}(t)+D_{i j m n} \varepsilon_{i j}(t) \kappa_{m n}(t)+\right. \\
+F_{i j m n r} \varepsilon_{i j}(t) \chi_{m n r}(t)+\frac{1}{2} B_{i j m n} \kappa_{i j}(t) \kappa_{m n}(t)+
\end{array}
$$




$$
\left.+G_{i j m n r} \kappa_{i j}(t) \chi_{m n r}(t)+\frac{1}{2} C_{i j k m n r} \chi_{i j k}(t) \chi_{m n r}(t)\right] d V .
$$

Proof. Let us fix an arbitrary $t \in(0, \infty)$. Then, with the usual calculations, we are led to the equality:

$$
\begin{array}{r}
\frac{\partial}{\partial t}\left(\rho \dot{\mathrm{u}}_{i}(\tau) \dot{\mathrm{u}}_{i}(2 t-\tau)+I_{j k} \dot{\phi}_{j m}(\tau) \dot{\phi}_{k m}(2 t-\tau)\right. \\
-c \theta(\tau) \theta(2 t-\tau))= \\
=\rho \ddot{\mathrm{u}}_{i}(\tau) \dot{\mathrm{u}}_{i}(2 t-\tau)+I_{j k} \ddot{\phi}_{j m}(\tau) \dot{\phi}_{k m}(2 t-\tau) \\
+c \theta(\tau) \dot{\theta}(2 t-\tau)--\rho \dot{\mathrm{u}}_{i}(\tau) \ddot{\mathrm{u}}_{i}(2 t-\tau) \\
+I_{j k} \dot{\phi}_{j m}(\tau) \ddot{\phi}_{k m}(2 t-\tau)-c \dot{\theta}(\tau) \theta(2 t-\tau) .
\end{array}
$$

Now, we consider the constitutive relations (4), the geometric equations (2), the relations of symmetry (8), and the equations of motion (9) so that we get the identity

$$
\begin{array}{r}
\frac{\partial}{\partial t}\left(\rho \dot{\mathrm{u}}_{i}(\tau) \dot{\mathrm{u}}_{i}(2 t-\tau)+I_{j k} \dot{\phi}_{j m}(\tau) \dot{\phi}_{k m}(2 t-\tau)\right. \\
-c \theta(\tau) \theta(2 t-\tau))==\left[\left(\tau_{i j}+\eta_{i j}\right)(\tau) \dot{\mathrm{u}}_{i}(2 t-\tau)\right. \\
-\left(\tau_{i j}+\eta_{i j}\right)(2 t-\tau) \dot{\mathrm{u}}_{j}(\tau)+(26) \\
+\mu_{i j k} \ddot{\phi}_{j k}(\tau) \dot{\phi}_{j k}(2 t-\tau)-\mu_{i j k} \ddot{\phi}_{j k}(2 t-\tau) \dot{\phi}_{j k}(\tau)- \\
\left.-\frac{1}{T_{0}} \theta(\tau) q_{i}(2 t-\tau)+\frac{1}{T_{0}} \theta(2 t-\tau) q_{i}(\tau)\right]_{, i}+F(\tau, 2 t-\tau),
\end{array}
$$

in which $F(.$, .) is the function introduced in (22).

Now the desired identity (24) will be obtained by integration in the above identity into $[0, t] \times D$. We must consider that the initial conditions are homogeneous and use the definition (13). As such, the demonstration of the proposition is finished.

In establishing the main results of our work, we will also use the utility of the two next enunciations.

Proposition 3. Let us consider that the array $\mathbf{u}=\left(\mathrm{u}_{i}, \phi_{i j}, \theta\right)$ satisfies the mixed problem $\mathcal{P}^{\prime}$. Then, the following equality is valid

$$
\begin{aligned}
& \int_{D}\left[\rho \mathrm{u}_{i}(t) \dot{\mathrm{u}}_{i}(t)+I_{j k} \phi_{j m}(t) \dot{\phi}_{k m}(t)\right. \\
& \left.-\frac{1}{2 T_{0}} K_{i j}\left(\int_{0}^{t} \theta(\tau) d \tau\right)_{, i}\left(\int_{0}^{t} \theta(\tau) d \tau\right)_{, j}\right] d V= \\
& =\int_{D}\left[\rho \mathrm{u}_{i}(0) \dot{\mathrm{u}}_{i}(0)+I_{j k} \phi_{j m}(0) \dot{\phi}_{k m}(0)\right] d V \\
& +\int_{0}^{t} \int_{D} \rho \eta(0) \theta(\tau) d V d \tau+ \\
& +\int_{0}^{t} \int_{D}\left[\rho \dot{\mathrm{u}}_{i}(t) \dot{\mathrm{u}}_{i}(t)+I_{j k} \dot{\phi}_{j m}(t) \dot{\phi}_{k m}(t)-2 \Psi(\tau)\right. \\
& \left.-c \theta^{2}(\tau)\right] d V d \tau,
\end{aligned}
$$

for all $t \in[0, \infty)$.

Proof. We first take into account the kinematic equations (2). Considering the constitutive equations (4) and the equations of motion $(9)_{1}$ and $(9)_{2}$, we get the equality:

$$
\frac{\partial}{\partial t}\left(\rho u_{i}(t) \dot{u}_{i}(t)+I_{j k} \phi_{j m}(t) \dot{\phi}_{k m}(t)\right)=
$$

$$
\begin{aligned}
& =\rho \dot{\mathrm{u}}_{i}(t) \dot{\mathrm{u}}_{i}(t)+I_{j k} \dot{\phi}_{j m}(t) \dot{\phi}_{k m}(t)+ \\
& +\left[\left(\tau_{i j}(t)+\eta_{i j}(t)\right) \mathrm{u}_{j}(t)+\mu_{i j k}(t) \phi_{j k}(t)\right]_{, i}- \\
& -\left[\left(\tau_{i j}(t)+\eta_{i j}(t)\right) \varepsilon_{i j}(t)+\eta_{i j}(t) \kappa_{i j}(t)+\mu_{i j k}(t) \chi_{i j k}(t)\right] .
\end{aligned}
$$

Considering the notation (13), we can restate the last parentheses of Equation (29) in the following form:

$$
\begin{aligned}
\tau_{i j}(t) \varepsilon_{i j}(t) & +\eta_{i j}(t) \kappa_{i j}(t)+\mu_{i j k}(t) \chi_{i j k}(t)= \\
= & \left(\frac{1}{2} c \theta^{2}(t)+\Psi(t)\right)+\left(\frac{1}{T_{0}} \theta(t) \int_{0}^{t} q_{i}(\tau) d \tau\right)_{, i}- \\
& \quad-\frac{1}{T_{0}} K_{i j}\left(\int_{0}^{t} \theta(\tau) d \tau\right)_{, i}\left(\int_{0}^{t} \theta(\tau) d \tau\right)_{, i}-\rho \eta(0) \theta(t) .
\end{aligned}
$$

We get the above expression of the entropy $\eta$ after we integrate the evolution equation $(19)_{3}$, satisfied by $\eta$, with respect to the variable $t$.

Finally, the equality (30) is integrated over $[0, t] \times D$. If we apply the divergence theorem and consider the boundary data (17), we arrive at the desired identity (29). As such, the proof of the proposition is finished.

Proposition 4. If the array $\left(u_{i}, \phi_{i j}, \theta\right)$ is a solution of the mixed backward in time problem $\mathcal{P}^{\prime}$, then we have the identity:

$$
\begin{aligned}
& 2 \int_{D}\left[\rho \mathrm{u}_{i}(t) \dot{\mathrm{u}}_{i}(t)+I_{j k} \phi_{j m}(t) \dot{\phi}_{k m}(t)\right. \\
& \left.-\frac{1}{2 T_{0}} K_{i j}\left(\int_{0}^{t} \theta(\tau) d \tau\right)_{, i}\left(\int_{0}^{t} \theta(\tau) d \tau\right)_{, j}\right] d V \\
& =\int_{D}\left[\rho \dot{\mathrm{u}}_{i}(0) \mathrm{u}_{i}(2 t)+I_{j k} \dot{\phi}_{j m}(0) \phi_{k m}(2 t)\right] d V+ \\
& +\int_{D}\left[\rho \mathrm{u}_{i}(0) \dot{\mathrm{u}}_{i}(2 t)+I_{j k} \phi_{j m}(0) \dot{\phi}_{k m}(2 t)\right] d V- \\
& -\int_{0}^{t} \int_{D} \rho \eta(0)[\theta(t+\tau)-\theta(t-\tau)] d V d \tau,
\end{aligned}
$$

for all $t \in[0, \infty)$.

Proof. We first consider the kinematic equations (2) and take into account the equations of motion $(9)_{1}$ and $(9)_{2}$. As such, we are led to the next equality

$$
\begin{array}{r}
\frac{\partial}{\partial t}\left[\rho\left(\dot{u}_{i}(t+\tau) u_{i}(t-\tau)+u_{i}(t+\tau) \dot{u}_{i}(t-\tau)\right)\right]+ \\
+\frac{\partial}{\partial t}\left[I_{j k}\left(\dot{\phi}_{j m}(t+\tau) \phi_{k m}(t-\tau)+\phi_{j m}(t+\tau) \dot{\phi}_{k m}(t-\tau)\right)\right]= \\
=\left[\left(\tau_{i j}+\eta_{i j}\right)(t+\tau) u_{i}(t-\tau)-\left(\tau_{i j}+\eta_{i j}\right)(t-\tau) u_{j}(t+\tau)+\right. \\
\left.+\mu_{i j k}(t+\tau) \phi_{j k}(t-\tau)-\mu_{i j k}(t-\tau) \phi_{j k}(t+\tau)\right]_{, i}- \\
-\left[\left(\tau_{i j}+\eta_{i j}\right)(t+\tau) \varepsilon_{i j}(t-\tau)-\left(\tau_{i j}+\eta_{i j}\right)(t-\tau) \varepsilon_{i j}(t+\tau)\right]- \\
-\left[\mu_{i j k}(t+\tau) \chi_{i j k}(t-\tau)-\mu_{i j k}(t-\tau) \chi_{j k}(t+\tau)\right] .
\end{array}
$$

Next, we consider the constitutive equations (4), so that by taking into account the equations of symmetry (8), the last two parentheses of the relation (32) receive the following expressions:

$$
\left[\left(\tau_{i j}+\eta_{i j}\right)(t+\tau) \varepsilon_{i j}(t-\tau)-\left(\tau_{i j}+\eta_{i j}\right)(t-\tau) \varepsilon_{i j}(t+\tau)\right]+
$$




$$
\begin{aligned}
& +\left[\mu_{i j k}(t+\tau) \chi_{i j k}(t-\tau)-\mu_{i j k}(t-\tau) \chi_{j k}(t+\tau)\right]= \\
& =\frac{1}{T_{0}}\left[\theta(t+\tau) \int_{0}^{t-\tau} q_{i}(s) d s-\theta(t-\tau) \int_{0}^{t+\tau} q_{i}(s) d s\right]_{, i}- \\
& -\frac{1}{T_{0}} K_{i j}\left[\left(\int_{0}^{t+\tau} \theta(s) d s\right)_{, i}\left(\int_{0}^{t-\tau} \theta(s) d s\right)_{, j}\right. \\
& \left.-\left(\int_{0}^{t+\tau} \theta(s) d s\right)\left(\int_{0}^{t-\tau} \theta(s) d s\right)_{, j}\right]- \\
& -\rho \eta(0)[\theta(t+\tau)-\theta(t-\tau)]
\end{aligned}
$$

We get the above expression of the entropy $\eta$ after we integrate the evolution equation $(19)_{3}$, satisfied by $\eta$, with respect to the variable $t$.

Finally, the equality (33) is integrated over $[0, t] \times D$. If we apply the divergence theorem and consider the boundary data (17), we arrive at the desired identity (31). As such, the proof of the proposition is finished.

A new useful identity will be obtained by combining the results from the identities (24) and (31), namely:

$$
\begin{aligned}
& 2 \int_{B}\left[\left(\rho \dot{\mathrm{u}}_{i}(t) \dot{\mathrm{u}}_{i}(t)+I_{j k} \dot{\phi}_{j m}(t) \dot{\phi}_{k m}(t)\right)-c \theta^{2}(t)\right] d V= \\
& =-2 \int_{D}\left[\rho \mathrm{u}_{i}(0) \dot{\mathrm{u}}_{i}(0)+I_{j k} \phi_{j m}(0) \dot{\phi}_{k m}(0)\right] d V+ \\
& +\int_{D}\left[\rho \dot{\mathrm{u}}_{i}(0) \mathrm{u}_{i}(2 t)+I_{j k} \dot{\phi}_{j m}(0) \phi_{k m}(2 t)\right] d V+ \\
& +\int_{D}\left[\rho \mathrm{u}_{i}(0) \dot{\mathrm{u}}_{i}(2 t)+I_{j k} \phi_{j m}(0) \dot{\phi}_{k m}(2 t)\right] d V+ \\
& -\int_{0}^{t} \int_{D} \rho \eta(0)[2 \theta(\tau)+\theta(t+\tau)-\theta(t-\tau)] d V d \tau,
\end{aligned}
$$

for all $t \in[0, \infty)$.

The utility of the previously proven equalities of integral type identities will result from their involvement in approaching the main results of our work. Our first main result is a theorem of uniqueness that will be established with regards to the solution of the backward in time problem. Then, the issue of locating solutions of the backward in time problem in time will be approached as a consequence of the result of uniqueness.

Theorem 1. The equations and conditions of the backward in time problem $\mathcal{P}^{\prime}$ can be satisfied by only one array $\mathbf{u}=\left(u_{i}, \phi_{i j}, \theta\right)$.

Proof. Suppose by absurdity, as usual, that our problem is satisfied by two arrays of the above form; that is, $\mathcal{P}^{\prime}$ would admit two different solutions. Clearly, since our problem $\mathcal{P}^{\prime}$ is a linear one, the difference of the respective two solutions is also a solution of the problem $\mathcal{P}^{\prime}$, but it corresponds to zero initial and boundary data. As such, it is enough to prove that the problem $\mathcal{P}^{\prime}$, for null data, admits the null solution. It is clear that for the difference of two solutions, the boundary and initial conditions become homogeneous.

The function $M$, defined in the following, is to simplify writing, and can be considered as a measure for the solutions of $\mathcal{P}^{\prime}$ :

$$
M(s)=\int_{D}\left[\frac{\varepsilon}{2}\left(\rho \dot{u}_{i}(t) \dot{u}_{i}(s)+I_{j k} \dot{\phi}_{j m}(s) \dot{\phi}_{k m}(s)\right)\right.
$$

$$
\left.+(\varepsilon+2) \Psi(s)+\frac{\varepsilon}{2} c \theta^{2}(s)\right],
$$

where $\varepsilon$ designates a sufficiently small positive constant.

It is not difficult to deduce that the above function $M$ is a positive one if we take into account the assumptions (a), (b), and (c). Based on the fact that the initial conditions are homogeneous, we can restate in a simpler form the identity (18):

$$
\begin{aligned}
& \int_{B}\left[\frac{1}{2}\left(\rho \dot{u}_{i}(t) \dot{u}_{i}(t)+I_{j k} \dot{\phi}_{j m}(t) \dot{\phi}_{k m}(t)\right)+\Psi(t)+\frac{1}{2} c \theta^{2}(t)\right] d V= \\
& =\int_{0}^{t} \int_{D} \frac{1}{T_{0}} K_{i j} \theta_{, i}(s) \theta_{, j}(s) d V d s, \forall t \in[0, \infty) .
\end{aligned}
$$

In a similar manner, we can give to the equality (21) the following simpler form:

$$
\begin{aligned}
& \int_{B}\left[\frac{1}{2}\left(\rho \dot{u}_{i}(s) \dot{u}_{i}(s)+I_{j k} \dot{\phi}_{j m}(s) \dot{\phi}_{k m}(s)\right)+\Psi(s)-\frac{1}{2} c \theta^{2}(s)\right] d V= \\
& =-\int_{0}^{s} \int_{D}\left\{\dot{u}_{i}(\tau)\left[\left(a_{i j}+b_{j i}\right) \theta(\tau)\right]_{, j}\right. \\
& +\dot{\phi}_{i j}(\tau)\left[c_{i j k} \theta(\tau)\right]_{, k}- \\
& \left.-b_{i j} \dot{\phi}_{i j}(\tau) \theta(\tau)+\frac{1}{T_{0}} K_{i j} \theta_{, i}(\tau) \theta_{, j}(\tau)\right\} d V d \tau \\
& \forall s \in[0, \infty) .
\end{aligned}
$$

With the help of the identities (36) and (37), we can restate the function $M$, defined in (35), as follows:

$$
\begin{aligned}
& M(t)=-\int_{0}^{t} \int_{D}\left\{2 \dot{u}_{i}(s)\left[\left(a_{i j}+b_{j i}\right) \theta(s)\right]_{, j}+\dot{\phi}_{i j}(s)\left[c_{i j k} \theta(s)\right]_{, k}-\right. \\
& \left.-b_{i j} \dot{\phi}_{i j}(s) \theta(s)+\frac{1-\varepsilon}{T_{0}} K_{i j} \theta_{, j}(s) \theta_{, i}(s)\right\} d V d s, \\
& \forall t \in[0, \infty) .
\end{aligned}
$$

If we use the derivative with respect to the time variable $t$ in (38), we get the following identity

$$
\begin{aligned}
& \frac{d M(t)}{d t}=-2 \int_{D}\left\{\dot{\mathrm{u}}_{i}(\tau)\left[\left(a_{i j}+b_{j i}\right) \theta(\tau)\right]_{, j}+\dot{\phi}_{i j}(\tau)\left[c_{i j k} \theta(\tau)\right]_{, k}-\right. \\
& \left.-b_{i j} \dot{\phi}_{i j}(\tau) \theta(\tau)+\frac{1-\varepsilon}{2 T_{0}} K_{i j} \theta_{, i}(\tau) \theta_{, j}(\tau)\right\} d V \\
& \forall t \in[0, \infty) .
\end{aligned}
$$

Next, in (39), we take into account the inequality of Schwarz and use a specific form of the arithmetic-geometric mean inequality so that we arrive at the next inequality

$$
\begin{aligned}
& \frac{d M(t)}{d t} \leq C_{1} \int_{D}\left[\rho \dot{\mathrm{u}}_{i}(t) \dot{\mathrm{u}}_{i}(t)+I_{j k} \dot{\phi}_{j m}(t) \dot{\phi}_{k m}(t)+c \theta^{2}(t)\right] d V+ \\
& +\frac{\delta-1+\varepsilon}{T_{0}} \int_{D} K_{i j} \theta_{, i}(\tau) \theta_{, j}(\tau) d V, \forall t \in[0, \infty) .
\end{aligned}
$$

Now, we remember that the energy density $\Psi$ is a quadratic form, positive definite. Also, we take into account hypothesis (c), use function $M$, defined in (35), and choose $\delta \leq 1-\varepsilon$. As such, from (40) we get the equality

$$
\frac{d M(t)}{d t} \leq \frac{C_{1}}{\varepsilon} \int_{D} \varepsilon\left[\rho \dot{\mathrm{u}}_{i}(t) \dot{\mathrm{u}}_{i}(t)+I_{j k} \dot{\phi}_{j m}(t) \dot{\phi}_{k m}(t)+c \theta^{2}(t)\right]
$$




$$
d V \leq \frac{C_{1}}{\varepsilon} M
$$

From this, we deduce that if the function $M(t)$ satisfies this inequality, it satisfies the following inequality:

$$
0 \leq M(t) \leq M(0) e^{C_{1} / \varepsilon}
$$

But, as we have already established, for the difference of the two solutions, the initial data are zero, so that, as a consequence, we have $M(0)=0$; as such, from (42), we deduce that:

$$
M(s)=0, \forall s \in[0, \infty)
$$

Using this statement and taking into account assumptions (a)-(c), we are led to the conclusion that the mixed problem admits only the null solution, i.e.

$$
u_{i}(s)=0, \phi_{i j}(s)=0, \theta(s)=0, \forall s \in[0, \infty)
$$

As such, Theorem 1 is proven.

For the final result of our study, we will approach the problem $\mathcal{P}^{*}$, which consists of the motion equations (9), the constitutive equations (4), the geometric equations (2), the initial restrictions (12), and the boundary homogeneous conditions (11). For this problem, we will establish that it is impossible to locate in time the solutions to this problem. In fact, it is shown that the solution with all null components, that is, the null solution, is the only solution of $\mathcal{P}^{*}$ that vanishes in the finite time.

Theorem 2. Assume that $\left(\mathrm{u}_{i}, \phi_{i j}, \theta\right)$ is a solution of the mixed problem $\mathcal{P}^{*}$, which decays in finite time $t_{0} \geq 0$,

$$
\mathrm{u}_{i}(t)=0, \phi_{i j}(t)=0, \theta(t)=0, \forall t \geq t_{0} .
$$

Then, the only solution that satisfies this condition is the null solution.

\section{REFERENCES}

1. Eringen AC. Theory of thermo-microstretch elastic solids. Int J Eng Sci. (1990) 28:1291-301. doi: 10.1016/0020-7225(90)90076-U

2. Eringen AC. Microcontinuum Field Theories. New York, NY: Springer (1999).

3. Marin M, Nicaise S. Existence and stability results for thermoelastic dipolar bodies with double porosity. Continuum Mech Therm. (2016) 28:1645-57. doi: 10.1007/s00161-016-0503-4

4. Marin M, Ellahi R, Chirila A. On solutions of Saint-Venant's problem for elastic dipolar bodies with voids. Carpathian J Math. (2017) 33:21932. Available online at: https://www.carpathian.cunbm.utcluj.ro/article/ solutions-saint-venants-problem-elastic-dipolar-bodies-voids/

5. Mindlin RD. Micro-structure in linear elasticity. Arch Ration Mech Anal. (1964) 16:51-78. doi: 10.1007/BF00248490

6. Green AE, Rivlin RS. Multipolar continuum mechanics. Arch Ration Mech Anal. (1964) 17:113-47. doi: 10.1007/BF00253051

7. Fried E, Gurtin ME. Thermomechanics of the interface between a body and its environment. Contin Mech Thermodyn. (2007) 19:253-71. doi: 10.1007/s00161-007-0053-x

8. Nunziato JW, Cowin SC. A nonlinear theory of materials with voids. Arch Ration Mech Anal. (1979) 72:175-201. doi: 10.1007/BF00249363
Proof. Using the way back in the previous procedure, we return to the backward in time problem defined on $\left(-\infty, t_{0}\right]$. Consider that this problem is defined by the equations of motion (9), the constitutive equations (4), the geometric equations (2), the null boundary data, and the homogeneous final restrictions:

$$
\begin{aligned}
& \mathrm{u}_{i}\left(x, t_{0}\right)=0, \dot{\mathrm{u}}_{i}\left(x, t_{0}\right)=0, \\
& \phi_{i j}\left(x, t_{0}\right)=0, \dot{\phi}_{i j}\left(x, t_{0}\right)=0, \theta\left(x, t_{0}\right)=0 .
\end{aligned}
$$

Based on Theorem 1, we deduce that the null solution is the only one with this property.

\section{CONCLUSIONS}

In our study, we formulated the linear backward in time problem for a thermoelastic body with a dipolar structure. In our first main result, we proved the uniqueness of the solution for this problem. Then, we approached the question of the possibility of locating the solution for this problem in time. So far, no study has addressed the problem of the localization in time of solutions for the mixed problem in the context of thermoelastic dipolar solids. We consider that, as suggested by a reviewer, it will be very useful for us to generalize the results of the present study in another study, in which we will replace the common derivative with a fractional one.

\section{DATA AVAILABILITY STATEMENT}

All datasets generated for this study are included in the article.

\section{AUTHOR CONTRIBUTIONS}

All authors listed have made a substantial, direct and intellectual contribution to the work, and approved it for publication. All authors have an equal and direct contribution, all have worked, elaborated and accepted the manuscript and are responsible for the results.

9. Cowin SC, Nunziato JW. Linear elastic materials with voids. J Elast. (1983) 13:125-47. doi: 10.1007/BF00041230

10. Goodman MA, Cowin SC. A continuum theory of granular material. Arch Ration Mech Anal. (1971) 44:249-66. doi: 10.1007/BF00284326

11. Marin M, Baleanu D, Vlase S. Effect of microtemperatures for micropolar thermoelastic bodies. Struct Eng Mech. (2017) 61:381-7. doi: 10.12989/sem.2017.61.3.381

12. Abbas IA. A GN model based upon two-temperature generalized thermoelastic theory in an unbounded medium with a spherical cavity. Appl Math Comput. (2014) 245:108-15. doi: 10.1016/j.amc.2014.07.059

13. Abbas IA. Eigenvalue approach for an unbounded medium with a spherical cavity based upon two-temperature generalized thermoelastic theory. J Mech Sci Technol. (2014) 28:4193-8. doi: 10.1007/s12206-014-0932-6

14. Othman MIA, Hasona WM, Abd-Elaziz EM. Effect of rotation on micropolar generalized thermoelasticity with two-temperatures using a dual-phase-lag model. Can J Phys. (2014) 92:149-58. doi: 10.1139/cjp-2013-0398

15. Marin M. Lagrange identity method for microstretch thermoelastic materials. J Math Anal Appl. (2010)363:275-86. doi: 10.1016/j.jmaa.2009.08.045

16. Marin M, Craciun EM. Uniqueness results for a boundary value problem in dipolar thermoelasticity to model composite materials, Compos Part B Eng. (2017) 126:27-37. doi: 10.1016/j.compositesb.2017.05.063 
17. Serrin J. The Initial Value Problem for the Navier-Stokes Equations. In: Nonlinear Problems (Proc. Sympos., Madison, Wis). Madison, WI: Univ. of Wisconsin Press. (1963). p. $69-98$

18. Ciarletta M, Scalia A. Some results in linear theory of thermomicrostretch elastic solids. Mecc. (2004) 39:191-206. doi: 10.1023/B:MECC.0000022843.48821.af

19. Knops RJ, Payne LE. On the stability of solutions of the navier-stokes equations backward in time. Arch Ration Mech Anal. (1968) 29:331-5. doi: 10.1007/BF00283897

20. Galdi GP, Straughan B. Stability of solutions of the navier-stokes equations backward in time. Arch Ration Mech Anal. (1988) 101:107-14.

21. Payne L, Straughan B. Improperly posed and nonstandard problems for parabolic partial differential equations. In: Eason RWOG, editor. Elasticity: Mathematical Methods and Applications, Philadelphia: Ellis Horwood (1990). p. 273-300.

22. Ames KA, Payne LE. Stabilizing solutions of the equations of dynamical linear thermoelasticity backward in time. Stab Appl Anal Contin Media. (1991) 1:243-60.

23. Ciarletta M. On the uniqueness and continuous dependence of solutions in dynamical thermoelasticity backward in time. J Therm Stresses. (2002) 25:969-84. doi: 10.1080/01495730290074423

24. Ciarletta M, Chirita S. Spatial Behavior in Linear Thermoelasticity Backward in Time. In: Chao CK, Lin CY, editors Proceedings of the Fourth International Congress on Thermal Stresses. Osaka (2001. p. 485-8.

25. Quintanilla R. Impossibility of localization in linear thermoelasticity with voids. Mech Res Commun. (2007) 34:522-7. doi: 10.1016/j.mechrescom.2007.08.004

26. Green AE, Naghdi PM. On undamped heat waves in an elastic solid. J Therm Stresses. (1992) 15:253-64. doi: 10.1080/01495739208946136

27. Green AE, Naghdi PM. Thermoelasticity without energy dissipation. J Elast. (1993) 31:189-208. doi: 10.1007/BF00044969

28. Iovane G, Passarella F. Saint-Venant's Principle in dynamic porous thermoelastic media with memory for heat flux. J Therm Stresses. (2004) 27:983-99. doi: 10.1080/01495730490498502

29. Passarella F, Tibullo V. Some results in linear theory of thermoelasticity backward in time for microstretch materials. J Therm Stresses. (2010) 33:55976. doi: 10.1080/01495731003772811
30. Marin M, Agarwal RP, Mahmoud SR. Nonsimple material problems addressed by the Lagrange's identity. Bound Value Probl. (2013) 2013:1-14. doi: 10.1186/1687-2770-2013-135

31. Marin M, Florea O. On temporal behaviour of solutions in thermoelasticity of porous micropolar bodies. An St. Univ Ovidius Constanta. (2014) 22:169-88. doi: 10.2478/auom-2014-0014

32. Marin M. Some estimates on vibrations in thermoelasticity of dipolar bodies. J Vib Control. (2010) 16:33-47. doi: 10.1177/1077546309103419

33. Marin M. On the minimum principle for dipolar materials with stretch. Nonlinear Anal Real World Appl. (2009) 10:1572-8. doi: 10.1016/j.nonrwa.2008.02.001

34. Quintanilla R, Straughan B. Energy bounds for some nonstandard problems in thermoelasticity. Proc $R$ Soc Lond A. (2005) 461:1-15. doi: 10.1098/rspa.2004.1381

35. Baleanu D, Sajjadi SS, Jajarmi A, Asad JH. New features of the fractional Euler-Lagrange equations for a physical system within non-singular derivative operator. Eur Phys J Plus. (2019) 134:1-10. doi: 10.1140/epjp/i2019-12561-x

36. Khiabani ED, Shiri B, Ghaffarzadeh H, Baleanu D. Visco-elastic dampers in structural buildings and numerical solution with spline collocation methods. J Appl Math Comput. (2019). doi: 10.1007/s12190-019-01307-5. [Epub ahead of print].

37. Alijani Z, Baleanu D, Shiri B, Wu G-C. Spline collocation methods for systems of fuzzy fractional differential equations, Chaos Solitons Fract. (2019) 131:109510. doi: 10.1016/j.chaos.2019.109510

Conflict of Interest: The authors declare that the research was conducted in the absence of any commercial or financial relationships that could be construed as a potential conflict of interest.

The handling editor declared a past co-authorship with one of the authors MM.

Copyright (c) 2020 Marin, Abbas, Vlase and Craciun. This is an open-access article distributed under the terms of the Creative Commons Attribution License (CC BY). The use, distribution or reproduction in other forums is permitted, provided the original author(s) and the copyright owner(s) are credited and that the original publication in this journal is cited, in accordance with accepted academic practice. No use, distribution or reproduction is permitted which does not comply with these terms. 